\title{
Variación de biomasa en un periodo de 21 años en un Bosque Atlántico del Alto Paraná (Paraguay)
}

\author{
Biomass variation over a 21 years period in an \\ Upper Parana Atlantic Forest, Paraguay
}

\author{
Victoria Rika Kubota ${ }^{1 *\left({ }^{(0}\right.}$, Rubén Darío Caballero-Cıonzález ${ }^{(}{ }^{(}$, \\ Aracery Elizabeth Fernández-Lomaquiz ${ }^{2}$
}

Kubota, V. R., Caballero-González, R. D. y Fernández-Lomaquiz, A. E. (2021). Variación de biomasa en un periodo de 21 años en un Bosque Atlántico del Alto Paraná (Paraguay). Colombia Forestal, 24(1), 60-70

Recepción: 16 de mayo 2020

\section{Resumen}

Se analizó la variación temporal de biomasa en un remanente del Bosque Atlántico del Alto Paraná en Paraguay, haciendo énfasis en la dinámica forestal y las clases diamétricas. Se estimó la biomasa total de los árboles con DAP $\geq 10 \mathrm{~cm}$, en una parcela permanente de 1 ha ubicada en la Reserva Natural Tati Yupi de Itaipu Binacional, empleando datos de mediciones de los años 1995, 2002, 2005, 2008, 2011 y 2016. En 21 años hubo un aumento de biomasa de $0.82 \mathrm{Mg}^{\mathrm{ha}}{ }^{-1} \cdot \mathrm{año}^{-1}$; sin embargo, la variación no fue uniforme entre las mediciones. En los periodos 1995-2002 y 2002-2005 se presen-

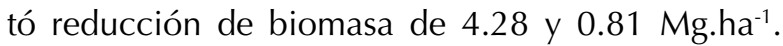
año ${ }^{-1}$, principalmente debido a la elevada mortandad de los árboles. Mientras que en los lapsos de 2005-2008, 2008-2011 y 2011-2016 hubo aumento de 6.00, 2.97 y $4.53 \mathrm{Mg} \cdot$ ha $^{-1}$. año ${ }^{-1}$, respectivamente. La variación fue más notable en las clases diamétricas inferiores.

Palabras clave: bosque subtropical, clase diamétrica, dinámica forestal, Reserva de Biosfera Itaipu, parcela permanente, Reserva Natural Tati Yupi
Aprobación: 21 de septiembre 2020

\begin{abstract}
The temporal variation of the tree biomass was analyzed in a remaining Upper Parana Atlantic Forest in Paraguay, focusing on forest dynamics and diameter classes. The total biomass of all individuals with $\mathrm{DBH} \geq 10 \mathrm{~cm}$ was estimated in a permanent plot of 1 ha located in the Tati Yupi Nature Reserve of Itaipu Binacional, using measurement data from 1995, 2002, 2005, 2008, 2011 and 2016. In 21 years, the biomass increased by $0.82 \mathrm{Mg}^{-h^{-1}}$. year $^{-1}$; however the variation was not uniform between measurements. In the period 1995 - 2002 and $2002-2005$, there was a reduction in total biomass of 4.28 and $0.81{\mathrm{Mg} . \mathrm{ha}^{-1} \text {.year }}^{-1}$, mainly due to high tree mortality. While in the period 2005 - 2008, 2008 - 2011 and 2011 - 2016, there was an increase in biomass of 6.00, 2.97 and $4.53{\mathrm{Mg} . \mathrm{ha}^{-1} \text {.year }}^{-1}$, respectively. The variation was more visible in lower diameter classes.
\end{abstract}

Key words: subtropical forest, diametric class, Forest dynamics, Itaipu Biosphere Reserve, Permanent plot, Tati Yupi Natural Reserve.

1 División de Áreas Protegidas, Dirección de Coordinación Ejecutiva, Itaipu Binacional, Paraguay.

2 Superintendencia de Obras y Desarrollo, Dirección de Coordinación Ejecutiva, Itaipu Binacional, Paraguay.

kubota@itaipu.gov.py. Autor de correspondencia. 


\section{INTRODUCCIÓN}

El estudio de la variación temporal de la biomasa forestal permite comprender mejor la participación de los bosques en el ciclo global de carbono, debido a que este elemento representa aproximadamente el 47 \% de la biomasa arbórea (McGroddy, Daufresne y Hedin, 2004; Pan et al., 2011). A medida que se evidencian los efectos del cambio climático, el interés mundial por la estimación y la conservación de la biomasa forestal incrementa (Pan et al., 2011). Esto se ve reflejado en la implementación de mecanismos como la reducción de emisiones por deforestación y degradación forestal en países en desarrollo (REDD+; Food and Agriculture Organization [FAO], 2020).

La biomasa en los bosques primarios tiende a mantenerse en equilibrio en el tiempo con pequeñas variaciones debido a disturbios naturales (Yamamoto, 2000). Estas fluctuaciones temporales a causa de la mortandad y el crecimiento de los árboles podrían ser consideradas como parte del funcionamiento normal de los bosques (Chave, Riéra y Dubois, 2001). Sin embargo, varios estudios demostraron que la biomasa de bosques tropicales está sufriendo cambios mayores con el paso de los años (Hoshizaki et al., 2004; Wigneron et al., 2020). Los fenómenos climáticos como El Niño y las sequías son algunas de las posibles causas de las transiciones extraordinarias de la biomasa forestal (Phillips y Lewis, 2016; Wigneron et al., 2020). Por otra parte, la biomasa tiende a incrementar a través del tiempo en los bosques en proceso de recuperación (Molas, 2016; Rodas, 2016). Estos estudios indican que aún existen vacíos de conocimiento sobre la dinámica de biomasa de los bosques.

En comparación con los bosques tropicales y los boreales, aún se conoce poco sobre la biomasa y la dinámica de los bosques subtropicales. Para la formulación y la puesta en funcionamiento de políticas de mitigación y adaptación al cambio climático, es necesario conocer el comportamiento de estos a través del tiempo (en lo que respecta a su biomasa) y analizarlo considerando el contexto global. Esto se puede lograr mediante el monitoreo de los bosques a través de las mediciones de parcelas permanentes que permiten identificar los cambios poblacionales y volumétricos en un área de estudio (Chave et al., 2001; Phillips et al., 2009). El monitoreo a largo plazo permite observar mejor las tendencias de la dinámica forestal.

El Bosque Atlántico del Alto Paraná (BAAPA) es una de las 15 ecorregiones que forma parte del complejo ecorregional denominado Bosque Atlántico y se encuentra en parte de Brasil, Paraguay y Argentina, con una superficie de 47120400 ha (Di Bitetti, Placci y Dietz, 2003). Originalmente, estuvo ocupado por bosques subtropicales semicaducifolios húmedos con la estructura más compleja y con mayor contenido de biomasa de Paraguay (Tortorelli, 1967). Pero, en los últimos siglos, el BAAPA fue explotado mediante la extracción de Ilex paraguariensis A. St.-Hil. y de maderas de especies de alto valor comercial y de grandes dimensiones, ocasionando la reducción de cantidad de árboles grandes en los bosques remanentes (Fleytas, 2007; Spichiger, Bertoni y Loizeau, 1992; Tortorelli, 1967). Adicional a esto, en las últimas décadas, extensas superficies del BAAPA fueron deforestadas y transformadas, principalmente, a cultivos y pasturas (De Sy et al., 2015). En Paraguay la cobertura forestal del BAAPA se redujo al $73 \%$ en 1973, al $24.9 \%$ en 2000 (Huang et al., 2007) y al $9 \%$ para el 2017 (Da Ponte, 2017). Esta fragmentación y degradación histórica influye en la cantidad y la dinámica de biomasa en los bosques remanentes (Facultad de Ciencias Agrarias, Universidad Nacional de Asunción, 2013). Sin embargo, aún es escaso el conocimiento de estas dinámicas a largo plazo en la región; por lo que este es el primer estudio con más de 20 años de monitoreo realizado en Paraguay.

La Reserva Natural Tati Yupi, administrada por la Itaipu Binacional, protege uno de los últimos remanentes del BAAPA en Paraguay. Fue creada en el año 1984 con el nombre de Refugio Biológico Tati Yupi y fue reconocida como área silvestre protegida mediante el Decreto Presidencial 7442 en el año 2017, adoptando la denominación de Reserva 
Natural Tati Yupi. Tiene 2245 ha, de las cuales 1672 ha corresponden a una formación boscosa que ha sido explotada en sus especies más valiosas antes de la creación del Refugio y que en la actualidad se encuentra en etapa de recuperación (Itaipu, 2016). De acuerdo con su composición arbórea (Caballero, 2014 y Vera, 2009), esta formación boscosa corresponde a bosques dominados por especies de la familia Lauraceae, así como especies de Cedrela fissilis Vell. y Chrysophyllum gonocarpum (Mart. \& Eichler) Engl., que se desarrollan en suelos bien drenados (Spichiger et al., 1992). El bosque de la Reserva Natural Tati Yupi tiene las características propias de los remanentes del BAAPA en proceso de recuperación.

El objetivo de este estudio fue analizar la variación temporal de la biomasa total arbórea en la Reserva Natural Tati Yupi, haciendo énfasis en la dinámica del bosque (mortandad, reducción volumétrica, crecimiento y reclutamiento de los árboles) y en las clases diamétricas de los individuos, considerando a esta reserva como una formación representativa del remanente del BAAPA en proceso de recuperación. La hipótesis planteada fue: en un periodo de 21 años hubo incremento de biomasa total arbórea debido al crecimiento y reclutamiento de los individuos, dándose la variación más notable en las clases diamétricas inferiores.

\section{MATERIALES Y MÉTODOS}

\section{Área de estudio}

El estudio se realizó en una parcela permanente de monitoreo instalada en la Reserva Natural Tati Yupi $\left(25^{\circ} 22^{\prime} 1.8^{\prime \prime}\right.$ latitud sur - 54³6'10.7' longitud oeste), la cual se encuentra en una zona periurbana del municipio de Hernandarias, departamento de Alto Paraná, Paraguay, y colinda con el embalse de Itaipu Binacional (figura 1). La altitud del terreno oscila entre los 220 y $260 \mathrm{~m}$ y presenta una temperatura media anual de $22{ }^{\circ} \mathrm{C}$ y una precipitación media anual de 1870 mm (Itaipu, 2016). El clima está clasificado, según Köppen, como templado lluvioso (Cfa) y, de acuerdo con el sistema de Thornthwaite, como Húmedo B2 (Itaipu, 2016). En la zona domina el suelo del orden Oxisol: de color pardo rojizo y textura limo arcillosa (Vera, 2009). La Reserva Natural Tati Yupi es una de las áreas núcleo de la Reserva de Biosfera de Itaipu, que fue reconocida por el programa Hombre y Biosfera de la Unesco en el año 2017.

Esta formación corresponde a un bosque subtropical semideciduo con especies latifoliadas (Vera, 2009). Algunas de las especies arbóreas representativas son: Diatenopteryx sorbifolia Radlk., Chrysophyllum gonocarpum (Mart. \& Eichler) Engl.,
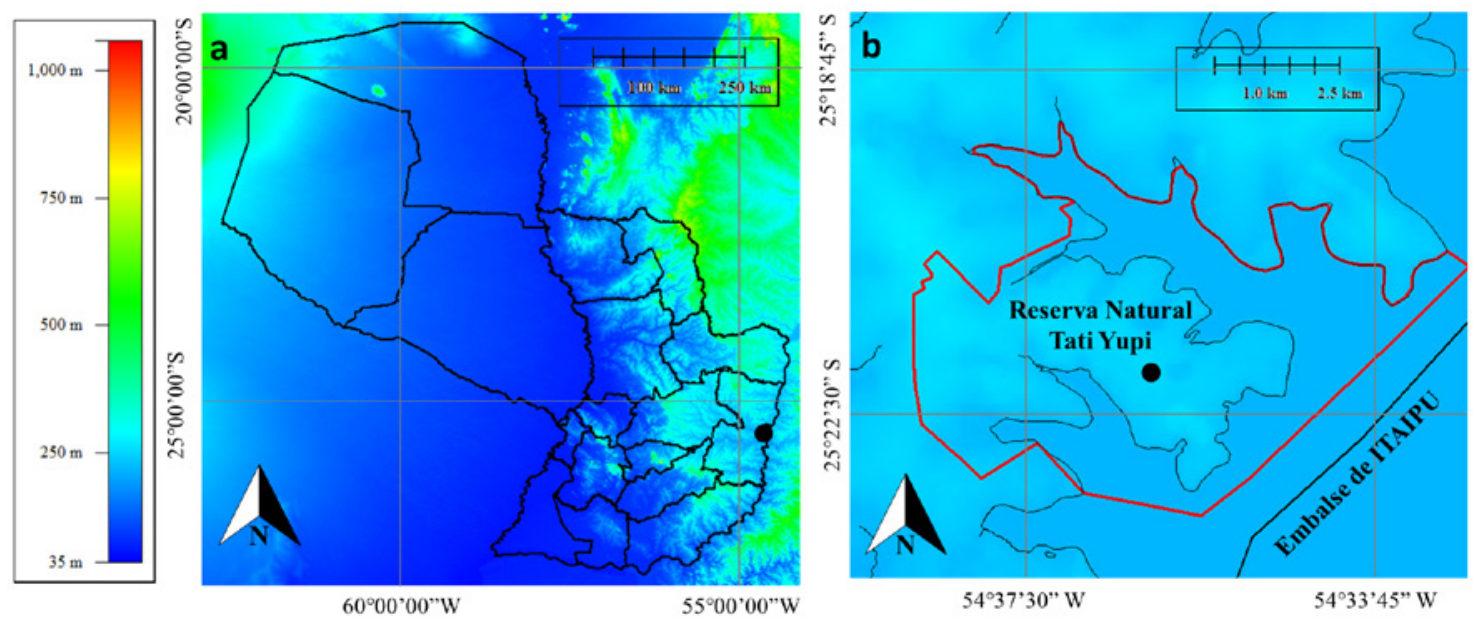

Figura 1a. Ubicación de la Reserva Natural Tati Yupi (punto negro) dentro del territorio paraguayo; b. Ubicación de la parcela permanente (punto negro) dentro de la Reserva Natural Tati Yupi (polígono rojo) y los principales cauces hídricos de la zona (líneas negras). 
Nectandra lanceolata Nees \& Mart., Cabralea canjerana (Vell.) Mart., Plinia rivularis (Cambess.) Rotman, Ocotea diospyrifolia (Meisn.) Mez, Cedrela fissilis Vell., Astronium fraxinifolium Schott, Holocalyx balansae Micheli, entre otras (Caballero, 2014). La cantidad de árboles por hectárea supera los 500 individuos que corresponden a 70 especies aproximadamente y la altura máxima del estrato arbóreo alcanza los 30 m (Vera, 2009).

\section{Muestreo y análisis de datos}

Fueron empleados datos de mediciones de una parcela permanente de $100 \times 100 \mathrm{~m}$ ( $1 \mathrm{ha}$ ) (Caballero, 2014), correspondientes a los años 1995, 2002, 2005, 2008, 2011 y 2016, lo que abarca un periodo de 21 años. Esta parcela está ubicada en un bosque alterado en proceso de recuperación y fue instalada evitando variaciones topográficas. Todos los árboles con diámetro a la altura del pecho (DAP) $\geq 10 \mathrm{~cm}$ están enumerados e identificados. En la primera medición fueron registrados datos de DAP y altura total de todos los individuos vivos; mientras que, para las mediciones posteriores, se utilizaron datos de individuos vivos, incluyendo a los reclutados que alcanzaron el DAP mínimo considerado; además, fueron registrados aquellos muertos después de la medición anterior. El DAP fue medido empleando cintas diamétricas, mientras que la altura fue estimada usando como referencia una vara altimétrica. Se calculó el diámetro cuadrático medio (DCM) de los fustes de los árboles bifurcados por debajo del DAP.

El monitoreo de 21 años fue posible debido a que tanto la metodología como los datos de medición depositados en la base de datos institucional son manejados por un equipo técnico designado a la evaluación y monitoreo de la biodiversidad de las áreas protegidas de Itaipu.

Se estimó la biomasa total (sumatoria de la biomasa aérea y subterránea) de los individuos registrados en cada una de las mediciones, empleando una ecuación alométrica (Sato et al., 2015).

$$
\mathrm{BT}=0.0505 \times\left(\mathrm{D}^{2} \mathrm{H}\right)^{0.9564}
$$

Donde,
BT: biomasa total $(\mathrm{kg})$
D: DAP $(\mathrm{cm})$ o DCM $(\mathrm{cm})$
$\mathrm{H}$ : altura total $(\mathrm{m})$

No se realizó descuento de biomasa en aqueIlos individuos con ramas rotas o aquellos con cavidades en los fustes. La biomasa de la parcela de cada medición fue estimada mediante la sumatoria de la biomasa total de todos los individuos vivos registrados en la ocasión y fue expresada en Mg.ha-1. Las diferencias entre las mediciones fueron consideradas como variación de biomasa total. El incremento de biomasa de la parcela debido al crecimiento y el reclutamiento de individuos fue considerado como ganancia; mientras que la variación de biomasa debido a la muerte y a la disminución de DAP o altura total fue considerada como reducción (transformación a necromasa).

Se realizó la comparación de biomasa total entre las mediciones. Además, se analizó la contribución de los cuatro factores de variación de biomasa y la variación por clase diamétrica.

\section{RESULTADOS}

\section{Variación de biomasa debido a la dinámica}

Durante 21 años la biomasa aumentó en 17.15 Mg.ha-1 (0.82 Mg.ha-1.año ${ }^{-1}$, figura 2a). Sin embargo, la variación no fue uniforme entre las mediciones. La biomasa total del bosque estudiado tuvo un descenso entre el año 1995 y el año 2005. Posteriormente, la biomasa fue aumentando gradualmente, hasta que en la última medición superó el valor registrado en el inicio. Así como la biomasa total, también se observó la variación en la cantidad de individuos inventariados, que fueron 509, 465, 477, 536, 567 y 567 para las siete mediciones. 
La mayor disminución de biomasa total se registró en el periodo 1995-2002, con una re-

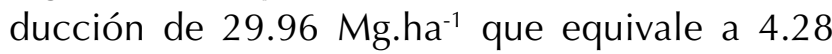
Mg.ha-1.año-1 (figura 2b). A partir del 2005 hasta el 2016 se registró un aumento gradual de biomasa total. En el periodo 2005-2008 se produjo el mayor incremento anual de biomasa $(6.00$ Mg.ha- $\left.{ }^{-1} \cdot a \tilde{n} o^{-1}\right)$.

En todos los periodos, la ganancia de la biomasa total debido al crecimiento de individuos fue superior al incremento por reclutamiento. Mientras que la reducción de biomasa total por la muerte de individuos fue superior a la reducción debido

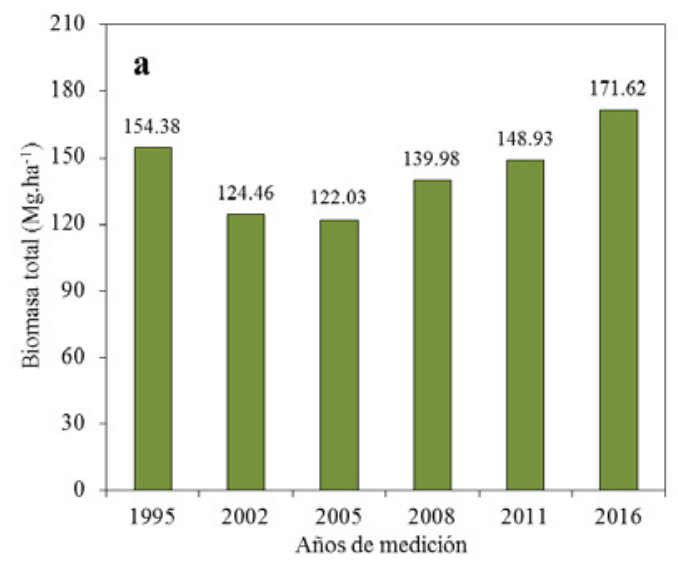

a la disminución del DAP o altura total, a excepción del periodo 2008-2011 (figuras 3a y 3b).

En el periodo 1995-2002 se registró la mayor cantidad de individuos muertos y con disminución de DAP o altura (tabla 1). Para el 2002, un total de 136 individuos, que equivale al $26.72 \%$ de árboles registrados en la medición del 1995, murieron ocasionando una reducción de biomasa total de $6.20 \mathrm{Mg} \cdot \mathrm{ha}^{-1}$. año ${ }^{-1}$; mientras que 98 individuos presentaron disminución de DAP o altura total causando una merma de biomasa de $1.52 \mathrm{Mg} \cdot \mathrm{ha}^{-1}$.año-1 (tabla 1, figuras 3a y $3 b)$. En este periodo también se observó la mayor cantidad de individuos reclutados (92).

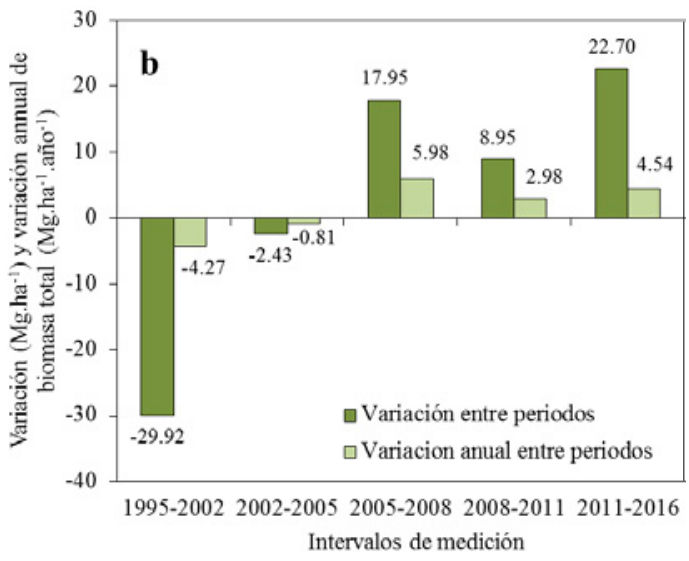

Figura 2a. Comparación de la biomasa total entre las mediciones; $\mathbf{b}$. Comparación de la variación de biomasa total y de la variación anual de biomasa total entre los periodos de medición.
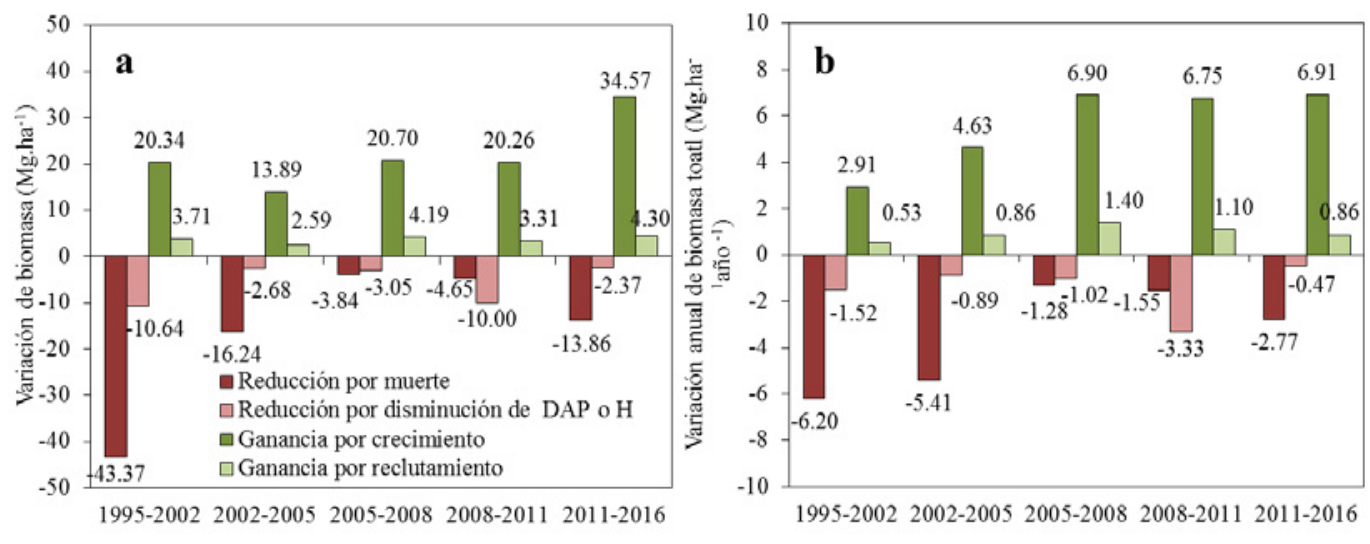

Intervalos de medición

Figura 3a. Comparación de la variación de biomasa total debido a la muerte, disminución de DAP o altura (H), crecimiento y reclutamiento de individuos entre periodos. b. Comparación de la variación anual de biomasa total debido a la muerte, disminución de DAP o altura $(\mathrm{H})$, crecimiento y reclutamiento de individuos entre periodos. 
Tabla 1. Número de árboles vivos, muertos, con disminución de DAP o altura $(\mathrm{H})$, reclutados y con crecimiento registrados en cada medición (ind.ha-1)

\begin{tabular}{cccccc}
\hline Condiciones & $\mathbf{1 9 9 5 - 2 0 0 2}$ & $\mathbf{2 0 0 2 - 2 0 0 5}$ & $\mathbf{2 0 0 5 - 2 0 0 8}$ & $\mathbf{2 0 0 8 - 2 0 1 1}$ & $\mathbf{2 0 1 1 - 2 0 1 6}$ \\
\hline Muertos & 136 & 60 & 25 & 41 & 74 \\
Con disminución de DAP o H & 98 & 44 & 73 & 90 & 40 \\
Reclutados & 92 & 72 & 84 & 72 & 74 \\
Con crecimiento & 260 & 320 & 371 & 403 & 434 \\
\hline
\end{tabular}

En los periodos 2002-2005 y 2005-2008 se observó la reducción gradual de la variación de biomasa total causada por la muerte de individuos (5.41 y $1.28 \mathrm{Mg} \mathrm{ha}^{-1}$.año ${ }^{-1}$ ) así como el número de árboles muertos (60 y 25; figura $3 a$, b y tabla 1 ). En los mismos periodos se registró el aumento gradual de la ganancia de biomasa total debido al crecimiento de los árboles (4.63 y 6.90 Mg.ha-1 ${ }^{-1}$ ano $^{-1}$ ), así como el número de individuos con crecimiento volumétrico $(320$ y 371). La ganancia de biomasa total por el reclutamiento de individuos también indicó un au-

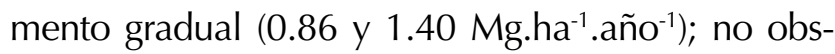
tante, el número de individuos reclutados fue inferior a los encontrados en el periodo 1995-2002 (72 y 84).

En los periodos 2008-2011 y 2011-2016 se observó un aumento en variación de biomasa total

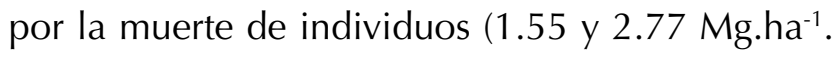
año ${ }^{-1}$ ) y de la cantidad de árboles muertos (41 y 74 , figura $3 a$, b y tabla 1). La ganancia de biomasa por crecimiento de individuos presentó valores similares entre los periodos (6.75 y $6.91{\mathrm{Mg} \cdot \mathrm{ha}^{-1} \text {.año }}^{-1}$ ), así como la cantidad de árboles con crecimiento (403 y 434).

\section{Variación de biomasa por clase diamétrica}

En todas las mediciones hubo mayor cantidad de individuos con diámetros inferiores (tabla 2). Por otra parte, el mayor valor de biomasa total fue registrado en las clases diamétricas intermedias (2029.9, 30-39.9 y 40-49.9cm). Se observó mayor ganancia de biomasa en las clases diamétricas inferiores, alcanzando un valor máximo de 3.95 y 1.63 Mg.ha-1.año-1 en la clase 10-19.9 y 20-29.9 $\mathrm{cm}$, respectivamente (tabla 3 ). A pesar de que las variaciones de biomasa fueron menores en las clases diamétricas superiores, la muerte de cada individuo de mayor dimensión tuvo gran influencia en la reducción de biomasa. En el periodo 19952002 la muerte de un individuo con DAP de 76.50

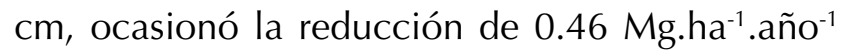
de biomasa (tabla 2 y 3 ).

Tabla 2. Distribución de biomasa total $\left(\mathrm{Mg}_{\mathrm{g}} \mathrm{ha}^{-1}\right)$ y cantidad de individuos (entre paréntesis) por clase diamétrica $(\mathrm{cm})$

\begin{tabular}{ccccccccccccccc}
\hline Clase diamétrica & \multicolumn{2}{c}{$\mathbf{1 9 9 5}$} & \multicolumn{2}{c}{$\mathbf{2 0 0 2}$} & \multicolumn{2}{c}{$\mathbf{2 0 0 5}$} & \multicolumn{2}{c}{$\mathbf{2 0 0 8}$} & \multicolumn{2}{c}{$\mathbf{2 0 1 1}$} & \multicolumn{2}{c}{$\mathbf{2 0 1 6}$} \\
\hline $\mathbf{1 0 - 1 9 . 9}$ & 22.98 & $(303)$ & 19.87 & $(292)$ & 20.33 & $(311)$ & 24.30 & $(351)$ & 28.03 & $(365)$ & 28.26 & $(347)$ \\
$\mathbf{2 0 - 2 9 . 9}$ & 31.28 & $(104)$ & 21.14 & $(80)$ & 21.42 & $(80)$ & 26.81 & $(98)$ & 30.38 & $(110)$ & 38.73 & $(129)$ \\
$\mathbf{3 0 - 3 9 . 9}$ & 36.84 & $(58)$ & 33.85 & $(54)$ & 31.85 & $(51)$ & 31.10 & $(48)$ & 30.95 & $(49)$ & 34.33 & $(50)$ \\
$\mathbf{4 0 - 4 9 . 9}$ & 34.94 & $(30)$ & 25.91 & $(26)$ & 24.32 & $(22)$ & 30.32 & $(26)$ & 27.30 & $(27)$ & 31.90 & $(24)$ \\
$\mathbf{5 0 - 5 9 . 9}$ & 19.48 & $(11)$ & 18.55 & $(11)$ & 18.39 & $(11)$ & 20.80 & $(11)$ & 23.09 & $(13)$ & 25.46 & $(13)$ \\
$\mathbf{6 0 - 6 9 . 9}$ & 2.02 & $(1)$ & 2.34 & $(1)$ & 2.53 & $(1)$ & 2.76 & $(1)$ & 4.88 & $(2)$ & 8.00 & $(3)$ \\
$\mathbf{7 0 - 7 9 . 9}$ & 6.87 & $(2)$ & 2.78 & $(1)$ & 3.18 & $(1)$ & - & - & - & - & - & - \\
$\mathbf{8 0 - 8 9 . 9}$ & - & - & - & - & - & - & 3.91 & $(1)$ & 4.29 & $(1)$ & 4.88 & $(1)$ \\
\hline
\end{tabular}


Tabla 3. Ganancia por crecimiento y por reclutamiento (Gan.), reducción por muerte y disminución de DAP o altura (Red.) y variación de biomasa total (Dif.) por clase diamétrica $(\mathrm{cm})$. Todos los valores en Mg.ha ${ }^{-1}$.año ${ }^{-1}$

\begin{tabular}{ccccccccccccccccc}
\hline \multirow{2}{*}{ Clase diamétrica } & \multicolumn{3}{c}{$\mathbf{1 9 9 5 - 2 0 0 2}$} & \multicolumn{2}{c}{$\mathbf{2 0 0 2 - 2 0 0 5}$} & \multicolumn{3}{c}{ 2005-2008 } & \multicolumn{3}{c}{ 2008-2011 } & \multicolumn{3}{c}{ 2011-2016 } \\
& Gan. & Red. & Dif. & Gan. & Red. & Dif. & Gan. & Red. & Dif. & Gan. & Red. & Dif. & Gan. Red. Dif. \\
\hline $\mathbf{1 0 - 1 9 . 9}$ & 1.45 & 0.99 & 0.46 & 2.45 & 0.85 & 1.60 & 3.67 & 0.45 & 3.21 & 3.95 & 0.87 & 3.08 & 3.12 & 0.84 & 2.28 \\
$\mathbf{2 0 - 2 9 . 9}$ & 0.73 & 1.57 & -0.84 & 1.28 & 0.79 & 0.49 & 1.26 & 0.46 & 0.81 & 1.53 & 0.87 & 0.65 & 1.63 & 0.64 & 0.99 \\
$\mathbf{3 0 - 3 9 . 9}$ & 0.68 & 1.37 & -0.70 & 0.79 & 1.84 & -1.05 & 1.34 & 1.19 & 0.16 & 1.10 & 0.90 & 0.20 & 1.33 & 0.61 & 0.72 \\
$\mathbf{4 0 - 4 9 . 9}$ & 0.44 & 1.75 & -1.31 & 0.57 & 1.75 & -1.18 & 0.89 & 0.19 & 0.69 & 0.79 & 1.70 & -0.91 & 0.82 & 0.78 & 0.04 \\
$\mathbf{5 0 - 5 9 . 9}$ & 0.10 & 1.44 & -1.34 & 0.21 & 1.08 & -0.87 & 0.81 & 0.01 & 0.81 & 0.29 & 0.53 & -0.24 & 0.69 & 0.38 & 0.31 \\
$\mathbf{6 0 - 6 9 . 9}$ & 0.05 & - & 0.05 & 0.06 & - & 0.06 & 0.08 & - & 0.08 & 0.07 & - & 0.07 & 0.07 & - & 0.07 \\
$\mathbf{7 0 - 7 9 . 9}$ & - & 0.58 & -0.58 & 0.13 & - & 0.13 & 0.24 & - & 0.24 & - & - & - & - & - & - \\
$\mathbf{8 0 - 8 9 . 9}$ & - & - & - & - & - & - & - & - & - & 0.13 & - & 0.13 & 0.12 & - & 0.12 \\
\hline
\end{tabular}

\section{DISCUSIÓN}

\section{Variación de biomasa}

La variación de número de individuos y de biomasa total observada en este estudio refleja la dinámica existente en el BAAPA. El comportamiento evidenciado en la parcela indica que posterior a la elevada pérdida de individuos o sus partes, como las ramas, hay una tendencia de incremento de regeneración y del crecimiento de los árboles remanentes. Este es un fenómeno explicado por Yamamoto (2000) y Lindner y Sattler (2012), quienes indican que la alteración de los bosques puede afectar la dinámica de tal forma que la perturbación podría ser apreciable en sus reservas de biomasa por largos periodos de tiempo.

El periodo de mayor reducción de biomasa total debido a la mortandad de árboles y disminución de DAP o altura total de este estudio (19952002) coincide con la ocurrencia del fenómeno del Niño extremadamente fuerte registrado entre el año 1997 a 1998, seguido de una prolongada La Niña que inició a mediados de 1998 y culminó al principio del 2001 (World Meteorological Organization [WMO], 2014). Wigneron et al. (2020) también encontraron reducción de biomasa forestal en los bosques tropicales de África, América y Asia como consecuencia del fenómeno El Niño registrado entre el 2015 a 2016. Por otra parte, Phillips et al. (2009) identificaron reducción de biomasa a causa de la sequía del año 2005 usando datos de 123 parcelas ubicadas en el Amazonas. No obstante, no se cuenta con suficientes evidencias para afirmar la relación entre la variación de biomasa total del bosque estudiado con los fenómenos climatológicos.

El aporte de los árboles con DAP elevado a la biomasa total de la parcela fue relevante a pesar de su poca abundancia. La cantidad de individuos con dimensiones mayores registrada en este estudio fue inferior a la reportada por Molas (2016), quien encontró cinco árboles con DAP > $80 \mathrm{~cm}$ en una parcela de $1 \mathrm{ha}$, teniendo uno de ellos un DAP $>100 \mathrm{~cm}$. Por otra parte, el DAP medio $(20.60 \pm 0.30 \mathrm{~cm})$ de la parcela estudiada determinado con los datos de todas las mediciones fue inferior al valor medio de las 13 parcelas instaladas en el BAAPA por Spichiger et al. (1992; $23.15 \pm 5.32 \mathrm{~cm})$.

Chave et al. (2003) reportaron una clara tendencia de incremento de biomasa en las clases diamétricas inferiores $(1-30 \mathrm{~cm})$ y una reducción en las clases superiores $(40-110 \mathrm{~cm})$. En este estudio la tendencia de la variación de biomasa por clase diamétrica fue similar pero no tan clara como la reportada por los citados autores. 


\section{Comparación con las tendencias globales}

Los estudios de la variación de biomasa realizados en la región indican una tendencia de incremento de la masa forestal a través del tiempo. No obstante, el incremento de biomasa encontrado en el pe-

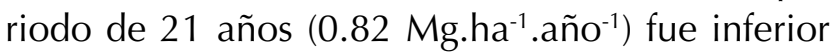
a los valores observados en estudios realizados en otras parcelas del BAAPA. Rodas (2016) encontró una ganancia biomasa total de $4.04 \mathrm{Mg} \cdot \mathrm{ha}^{-1} \cdot$ año $^{-1}$ en un bosque secundario de 29 años de recuperación. Mientras que Molas (2016) observó un aumento de $2.35 \mathrm{Mg} \cdot \mathrm{ha}^{-1} \cdot$ año $^{-1}$ en un bosque alterado del BAAPA. El bajo incremento observado en el presente estudio se debió a la elevada mortandad que tuvo lugar en las primeras mediciones; posterior al año 2005, el incremento de biomasa fue similar a los encontrados por Rodas (2016) y Molas (2016).

Tendencias similares fueron observadas en estudios a largo plazo realizados en bosques del neotrópico. Chave et al. (2001) monitorearon la biomasa aérea en un bosque ubicado en Guayana Francesa registrando un aumento de 1.9 Mg.ha-1.año-1 y $0.7 \mathrm{Mg} \cdot \mathrm{ha}^{-1} \cdot \mathrm{año}^{-1}$ en dos sitios estudiados durante el periodo 1981-1991. Chave et al. (2003) determinaron que el aumento de biomasa aérea en una parcela de 50 ha de un bosque tropical en Panamá durante el periodo 1985-2000 fue de $0.21 \mathrm{Mg} \cdot \mathrm{ha}^{-1}$.año ${ }^{-1}$. Phillips y Lewis (2009) estimaron un incremento de biomasa aérea de $0.89 \mathrm{Mg} \mathrm{ha}^{-1}$.año ${ }^{-1}$ usando datos de 123 parcelas ubicadas en el Amazonas (1991-2005). Baker et al. (2004) observaron un incremento medio de biomasa aérea de $1.22{\mathrm{Mg} . h \mathrm{H}^{-1} \text {.año }}^{-1}$ en 59 parcelas instaladas en el Amazonas que fueron medidas en el periodo 1979-2001. En el continente africano también fue observado un incremento de biomasa forestal. Lewis et al. (2009) analizaron datos de 79 parcelas instaladas en 10 países africanos y encontraron un incremento de biomasa aérea de aproximadamente $1.26 \mathrm{Mg} \cdot \mathrm{ha}^{-1} \cdot \mathrm{anno}^{-1}$ (0.63 MgC.ha ${ }^{-1}$.año-1 $)$ en el lapso 1968-2007.
En lo concerniente a la región paleotropical, Hoshizaki et al. (2004) encontraron una disminución de la biomasa aérea equivalente a 7.10 Mg.ha-1.año-1 en los bosques de Malasia (19941998) debido, principalmente, a la muerte de árboles grandes. Este resultado contrasta con las tendencias reportadas por autores ya citados, quienes analizaron datos provenientes de otras regiones, y con las observaciones del presente estudio.

\section{Desafíos para futuros estudios}

Es necesario generar más información sobre la variación temporal de biomasa de los remanentes del BAAPA para lograr un conocimiento integral sobre la participación de estos bosques en el ciclo global de carbono.

En este estudio no se tuvo en cuenta el efecto de los árboles con ramas rotas y con fustes ahuecados sobre la biomasa total. Monda et al. (2015) reportaron que la aplicación de ecuaciones alométricas específicas para árboles con cavidades tuvo una influencia importante sobre el valor final de la biomasa de un bosque del sureste asiático, debido a que las cavidades son más comunes en los árboles de mayor dimensión. Considerando que la abundancia de los árboles ahuecados podría variar entre sitios, se requiere de un análisis minucioso para determinar la necesidad de realizar los ajustes correspondientes para estudios de biomasa que sean llevados a cabo en el BAAPA.

\section{CONCLUSIONES}

La hipótesis planteada para el estudio fue aceptada. Hubo un aumento de biomasa total en el transcurso de 21 años. No obstante, la variación no fue uniforme entre las mediciones. Los principales factores que perturbaron la biomasa total fueron la muerte y el crecimiento de los individuos. La elevada cantidad de individuos muertos causó la disminución de biomasa total entre la primera y 
segunda medición. Posteriormente, se observó un aumento gradual de la biomasa total debido a la reducción de la cantidad de individuos muertos y al incremento por crecimiento de árboles. La influencia de la reducción de DAP o altura y el reclutamiento de individuos fueron menos relevantes.

En todas las mediciones se encontraron mayores variaciones de biomasa en las clases diamétricas inferiores. Por otra parte, la muerte de individuos con dimensiones superiores causó reducciones importantes de biomasa debido a su gran volumen, a pesar de que la cantidad de estos individuos fue baja.

Este es el primer estudio de dinámica de biomasa a largo plazo realizado en Paraguay y servirá de referencia para los bosques remanentes del BAAPA en proceso de recuperación. El incremento de biomasa observado sugiere que los remanentes del BAAPA podrían estar cumpliendo una función positiva en la mitigación de cambio climático. Es necesario generar más información sobre esta dinámica para lograr un conocimiento integral sobre la participación del BAAPA en el ciclo global de carbono.

\section{AGRADECIMIENTOS}

La instalación y las mediciones de la parcela permanente fueron realizadas con recursos de la Itaipu Binacional. Se agradece a todas las personas que participaron y apoyaron las mediciones de la parcela estudiada a lo largo de 21 años. En especial a: Carlos Giménez Facetti (†), Amancio MaIlorquín, Nicolás Coronel, Vicente López, Edgar Domínguez, Rubén Rodríguez, Germán Mendoza $(\mathbf{t})$, Néstor Ayala (†), Guillermo Caballero, Antonio Lomaquis, Francisco Calonga y Raúl Ortega, quienes iniciaron este monitoreo al instalar la parcela y realizar la primera medición.

\section{CONFLICTO DE INTERESES}

Los autores declaran no tener conflicto de intereses

\section{CONTRIBUCIÓN POR AUTORES}

R.D.C.G. realizó el registro de datos en terreno en los años 2002, 2005, 2008 y 2016. A.E.F.L. participó en el registro de datos en terreno en el año 2016. V.R.K. participó en el registro en terreno en el año 2016 y analizó los datos. Todos los autores contribuyeron a la discusión y participaron en la redacción del manuscrito.

\section{REFERENCIAS}

Baker, T. R., Phillips, O. L., Malhi, Y., Almeida, S., Arroyo, L., Di Fiore, A., Erwin, T., Higuchi, N., Killen, T. J., Laurance, S. G., Laurance, W. F., Lewie, S. L., Monteagudo, A., Neil, D. A., Núñez Vargas, P., Pitman, N. C. A., Silva, J. N. M. y Vásquez Martínez, R. (2004). Increasing biomass in Amazonian forest plots. Philosophical Transactions of The Royal Society, 359, 353-365. https://doi.org/10.1098/rstb.2003.1422

Caballero, R. (2014). Análisis estructural y dinámico de la parcela permanente de medición y monitoreo de la biodiversidad del Refugio Biológico Tati Yupi: fitosociología forestal, análisis de datos de campo. Biota, 16.

Chave, J., Condit, R., Lao, S., Caspersen, J., Foster, R. y Hubbell, S. (2003). Spatial and temporal variation of biomass in a tropical forest: results from a large census plot in Panama. Journal of Ecology, 91, 240-252. https://doi.org/10.1046/j.1365-2745.2003.00757.x

Chave, J., Riéra, B. y Dubois, M. A. (2001). Estimation of biomass in a neotropical forest of French Guiana: spatial and temporal variability. Journal of Tropical Ecology, 17, 79-96. https://doi.org/10.1017/S0266467401001055

Da Ponte, E., Mack, B., Wohlfart, C., Rodas, O., Fleckenstein, M., Oppelt, N., Dech, S. y Kuenzer C. (2017). Assessing forestcover dynamics and forestperception in the Atlantic Forest of Paraguay, combining remote sensing and household level data. Forests, 8(389). https://doi.org/10.3390/f8100389 
De Sy, V., Herold, M., Achard, F., Beuchle, R., Clevers, J. G. P. W., Lindquist, E. y Verchot, L. (2015). Land use patterns and related carbon losses following deforestation in South America. Environmental Research Letters, 10, 124004. https://doi.org/10.1088/1748-9326/10/12/124004

Di Bitetti, M., Placci, G. y Dietz, L. (2003). A biodiversity vision for the Upper Paraná Atlantic Forest ecoregion: designing a biodiversity conservation landscape and setting priorities for conservation action. World Wildlife Fund.

Facultad de Ciencias Agracias, Universidad Nacional de Asunción (2013). Mapa de stock de carbono bosque atlántico del alto Paraná. https://globallandusechange.org/wp-content/ uploads/2017/10/Reporte.-2014.-Carrera-Ingeneria-Forestal-UNA-Desarrollo-del-estudio-de-linea-de-base-para-el-sitio-piloto-Bosque-Atlantico-Alto-Parana.pdf

Fleytas, M. C. (2007). Cambios en el paisaje: evolución de la cobertura vegetal en el Paraguay. En D. A. Salas-Dueñas y J. F. Facetti (eds.), Biodiversidad del Paraguay: una aproximación a sus realidades (pp. 77-87). Fundación Moisés Bertoni, USAID, GEF/ $\mathrm{BM}$

Food and Agriculture Organization (FAO) (2020). REDD+: Reducción de las emisiones derivadas de la deforestación y la degradación de los bosques. http://www.fao.org/redd/overview/es/

Hoshizaki, K., Niiyama, K., Kimura, K., Yamashita, T., Bekku, Y., Okuda, T., Quah, E. y Supardi, N. (2004). Temporal and spatial variation of forest biomass in relation to stand dynamics in a mature, lowland tropical rainforest, Malaysia. Ecological Research, 19, 357-363. https://doi.org/10.1111/j.1440-1703.2004.00645.x.

Huang, C., Kinm, S., Alstatt, A., Townshend, J. R. G., Davis, P., Song, K., Tucker, C. J., Rodas, O., Yanosky, A., Clay, R. y Musinsky, J. (2007). Rapid loss of Paraguay's Atlantic forest and the status of protected area: a Landsat assessment. Remote Sensing of Environment, 106(4), 460-466. https://doi.org/10.1016/j.rse.2006.09.016
Itaipu Binacional (2016). Plan de manejo 2017-2021 de la Reserva Natural Tati Yupi. Editorial Fotosíntesis.

Lewis S. L., López-González G., Sonke B., Affum-Baffo K., Baker T. R., Ojo L. O., Phillips O. L., Reitsma J., White L., Comiskey J. A., Ewango C., Feldpausch T., Hamilton A. C., Gloor E., Hart T., Hladik A., Kamdem M. N. D., Lloyd J., Lovett J., Makana J. R., [...] y Woll, H. (2009). Increasing carbon storage in intact African tropical forests. Nature, 477, 1003-1006. https://doi.org/10.1038/nature07771

Lindner, A. y Sattler, D. (2012). Biomass estimations in forests of different disturbance history in the Atlantic Forest of Rio de Janeiro, Brazil. New Forests, 43, 287-301. https://doi.org.10.1007/s11056-011-9281-9

McGroddy, M. E., Daufresne, T. y Hedin, L.O. (2004). Scaling of C: N: P stoichiometry in forests worldwide: Implications of terrestrial Redfield-type ratios. Ecology, 85, 2390-2401. https://doi.org/10.1890/03-0351

Molas, C. (2016). Variación temporal de la biomasa y carbono de un bosque del BAAPA en el periodo 2010-2015, Reserva Natural del Bosque Mbaracayú, Departamento de Canindeyú (tesis de grado, Ingeniería Ambiental). Universidad Nacional de Asunción, San Lorenzo, Paraguay.

Monda, Y., Kiyono, Y., Melling, L., Damian, Ch. y Chaddy, A. (2015). Allometric equations considering the influence of hollow trees: A case study for tropical peat swamp forest in Sarawak. Tropics, 24(1), 11-22. https://doi.org/10.3759/tropics.24.11

Pan, Y., Birdsey, A. R., Fang, J., Houghton, R., Kauppi, P. E., Kurz, W. A-. Phillips, P. L., Shvidenko, A., Lewis, S. L., Canadell, J. G., Ciais, P., Jackson, R. B., Pacala, S. W., McGuire, A. D., Piao, S., Rautiainen, A., Sitch, S. y Hayes, D. (2011). A large and persistent carbon sink in the world's forests. Science, 333, 988-993. https://doi.org/10.1126/science.1201609

Phillips O. L., Aragao L. E. O. C, Lewis, S. L, Fisher, J. B., Lloyd, J., López-González, G., Malhi, Y., Monteagudo, A., Peacock, J., Quesada, C. A., van der Heijden, G., Almeida, S., Amaral, I., Arroyo, L., Aymard, G., Baker, T. R., 
Banki, O., Blanc, L., Bonal, D., Brando, P., [...] y Torres-Lezama, A. (2009). Drought sensitivity of the Amazon rainforest. Science, 323, 1344-1347. https://doi.org.10.1126/science.1164033

Phillips, O. y Lewis, S. (2016). Recent changes in tropical forest biomass and dynamics. En L. Nagy, B. R. Forsberg y P. Artaxo (eds.), Interactions between biosphere, atmosphere and human land use in the amazon basin (pp. 191-224). Springer. https://doi.org/10.1007/978-3-662-49902-3_10

Rodas Amarilla, G. D. (2016). Variación temporal de la biomasa y carbono de un bosque secundario del BAAPA, periodo 2009-2016, Reserva Natural del Bosque Mbaracayu, departamento de Canindeyú (tesis de grado, Ingeniería Forestal). Universidad Nacional de Asunción, San Lorenzo, Paraguay.

Sato, T., Saito, M., Ramírez, D., Pérez de Molas, L.F., Toriyama, J., Monda, Y., Kiyono, Y., Herebia, E., Dubie, N., Duré Vera, E., Ramírez Ortega, J. D. y Vera de Ortiz, M. (2015). Development of allometric equations for tree biomass forest ecosystems in Paraguay. Japan Agricultural Research Quarterly, 49(3), 281-291.
Spichiger, R., Bertoni, B. y Loizeau, P. A. (1992). The forest of the paraguayan Alto Paraná. Candollea, $47(2), 219-250$.

Tortorelli, L. A. (1967). Formaciones forestales y maderas del Paraguay. Instituto Forestal Latino-Americano de Investigación y Capacitación, 24, 3-34.

Vera Monge, V. R. (2009). Comparação de associações vegetais sobre diferentes tipos de solos na área de influência da Represa Itaipu, para reconhecimento de espécies apropriadas para restauração ecológica (tesis de doctorado en Recursos Forestales). Universidad de San Pablo, Brasil.

Wigneron, J. P., Fan, L., Ciais, P., Bastos, A., Brandt, M., Chave, J., Saatchi, S., Baccini, A. y Fensholt, R. (2020). Tropical forests did not recover from the strong 2015-2016 El Niño event. Science Advances, 6(6), eaay4603. https://doi.org/10.1126/sciadv.aay4603

World Meteorological Organization (WMO) (2014). El Niño: Southern Oscillation. Ginebra: WMO.

Yamamoto, S. (2000). Forest gap dynamics and tree regeneration. Journal of Forestry Research, 5, 223-229. https://doi.org/10.1007/BF02767114

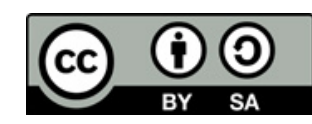

Colombia Forestal •ISSN 0120-0739 • e-ISSN 2256-201X • Bogotá-Colombia • Vol. 24 No. 1 • Enero-Junio de 2021 • pp. 60-70 [ 70 ] 